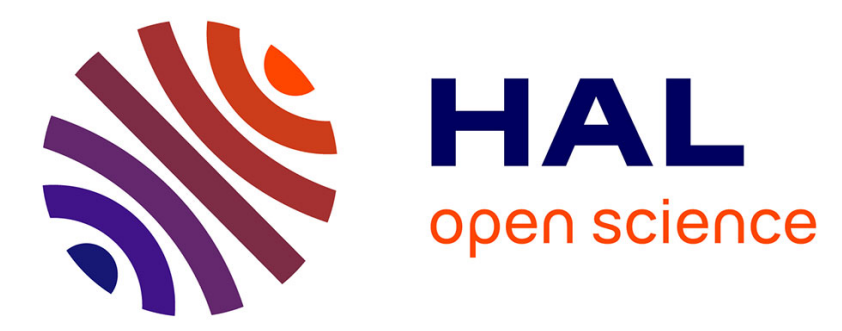

\title{
Exploring mobile news reading interactions for news app personalisation
}

Marios Constantinides, John Dowell, David Johnson, Sylvain Malacria

\section{To cite this version:}

Marios Constantinides, John Dowell, David Johnson, Sylvain Malacria. Exploring mobile news reading interactions for news app personalisation. 17th International Conference on HumanComputer Interaction with Mobile Devices and Services, Aug 2015, Copenhagen, Denmark. pp.6, $10.1145 / 2785830.2785860$. hal-01252631

\section{HAL Id: hal-01252631 \\ https://inria.hal.science/hal-01252631}

Submitted on 13 Jan 2016

HAL is a multi-disciplinary open access archive for the deposit and dissemination of scientific research documents, whether they are published or not. The documents may come from teaching and research institutions in France or abroad, or from public or private research centers.
L'archive ouverte pluridisciplinaire HAL, est destinée au dépôt et à la diffusion de documents scientifiques de niveau recherche, publiés ou non, émanant des établissements d'enseignement et de recherche français ou étrangers, des laboratoires publics ou privés.

\section{다)(1) $(5$}

Distributed under a Creative Commons Attribution - NonCommercial| 4.0 International 


\title{
Exploring mobile news reading interactions for news app personalisation
}

\author{
Marios Constantinides ${ }^{1}$, John Dowell ${ }^{1}$, David Johnson ${ }^{1}$, Sylvain Malacria ${ }^{1,2}$ \\ ${ }^{1}$ University College London, \\ London, United Kingdom \\ $\{$ m.constantinides, j.dowell\}@cs.ucl.ac.uk \\ david.johnson.11@ucl.ac.uk \\ ${ }^{2}$ Inria Lille \\ Lille, France \\ sylvain.malacria@inria.fr
}

\begin{abstract}
As news is increasingly accessed on smartphones and tablets, the need for personalising news app interactions is apparent. We report a series of three studies addressing key issues in the development of adaptive news app interfaces. We first surveyed users' news reading preferences and behaviours; analysis revealed three primary types of reader. We then implemented and deployed an Android news app that logs users' interactions with the app. We used the logs to train a classifier and showed that it is able to reliably recognise a user according to their reader type. Finally we evaluated alternative, adaptive user interfaces for each reader type. The evaluation demonstrates the differential benefit of the adaptation for different users of the news app and the feasibility of adaptive interfaces for news apps.
\end{abstract}

\section{Author Keywords}

Mobile news reading; Personalisation; Implicit sampling; Adaptive mobile User Interfaces;

\section{ACM Classification Keywords}

H.5.m. Information interfaces and presentation (e.g., HCI): Miscellaneous.

\section{INTRODUCTION}

Mobile app ecosystems are transforming patterns of news consumption. Until quite recently, reading the news was a niche use for smartphones [12], mostly for when users were 'on the go'; now however, two in every three users of mobile devices in the US regularly access news and as many as one in five read in-depth news articles daily [2]; a similar picture is found in the UK [1]. This growth in mobile news access continues the migration of news consumers to the Internet.

Permission to make digital or hard copies of all or part of this work for personal or classroom use is granted without fee provided that copies are not made or distributed for profit or commercial advantage and that copies bear this notice and the full citation on the first page. Copyrights for components of this work owned by others than ACM must be honored. Abstracting with credit is permitted. To copy otherwise, or republish, to post on servers or to redistribute to lists, requires prior specific permission and/or a fee. Request permissions from Permissions@acm.org. MobileHCI'15, August 25 - 28, 2015, Copenhagen, Denmark (C) 2015 ACM. ISBN 978-1-4503-3652-9/15/08...\$15.00

DOI: http://dx.doi.org/10.1145/2785830.2785860

Mobile news access perfectly complements the continuously updating, 24-hour nature of digital news services. But if users are now never out of range of the news, they need more than ever for that access to be adaptive and personalised. Personalised news services are already able to help people find news that is relevant to them, to recommend the right news to the right users, and to help users keep abreast of news by aggregation over multiple sources. This adaptivity is achieved through several methods [5] including: news content personalisation by pushing filtered articles predicted to match the user's interests; adaptive news browsing by changing the order of news categories; contextual news access by offering users access to additional information related to the news they are reading; and news aggregation, by automatically identifying main news topics emerging from multiple sources. This previous work on adaptivity in digital news access has focused on recommendation of news content. But, adaptation of the way people interact with news services has not been investigated.

Personalisation of news access clearly needs to extend beyond 'what' content users access to 'how' they access it, as evident in the abundance of mobile news apps offering personalisation features. For example, Inside.com Breaking News allows users to select news topics to follow and then provides 300-character summaries of relevant stories along with links to the original sources. Another example is Newsbeat, again an aggregator but one that creates 'personalised radio news bulletins'. Users select their preferred text news sources from which stories are pulled each day, summaries created, then news podcasts created using text-to-voice technology. A third example is Flipboard, which uses the metaphor of a 'personal magazine' to present articles from conventional news providers as well as social media updates, and RSS feeds. Users curate and share their own mini-magazines within the app, drawing in stories on their preferred topics.

The personalisation of news app interaction in these examples is achieved through making the interface adaptable. Adaptive news interfaces that 'automatically' adapt to the way the user reads the news in particular contexts are not found, other than in re-ordering menus of headlines to take account of previous reading choices. This adaptation could be far more extensive, for example, to take 
account of users' idiosyncratic patterns of browsing news headlines or the different ways in which different users read news articles $[8,12]$.

Adaptive personalisation relies on constructing and exploiting an individual user profile to deliver a tailored version of the user interface [9]. A user profile is a model of the user that the system learns through interaction with the user. The construction of a user profile can be based on explicit or implicit information gathering approaches. The former consists of information provided directly by the user such as by forms and questionnaires; although data captured this way will have a greater reliability, the disruption to the user can be significant. Recent studies attempting to compare both methods have found implicit data capture to be preferred by users [7].

User profiling has been demonstrated with news service applications. Billsus and Pazzani [4] developed the news recommendation system NewsDude to recommend news articles for desktop users. They used supervised machine learning methods in the form of nearest-neighbor algorithms to model short-term interests, and a naive Bayes classifier for long-term interests. Carreira et al. [6] used interaction $\operatorname{logs}$ of mobile users of news services to implicitly capture user profiles as the basis for recommending articles of interest. Their prototype news application logged aspects of users' reading behaviour such as reading duration, estimated number of lines read, estimated reading speed, etc. However, they logged interactions with news services on PDAs having much less advanced capabilities (neither 3G nor high resolution screen). Recently Tavakolifard et al. [11] proposed a news content recommendation system (including the mobile app) that efficiently delivers "tailored news in the palm of your hand". No studies have attempted to log interactions for the purpose of personalising the interface as opposed to personalising the new content.

Interaction data capture with smartphones has been demonstrated in other studies but not in relation to news consumption. Oulasvirta et al. [10] used logs of smartphone interactions to examine users' habits, in particular their habitual checking of their smartphone state and notifications. Woerndl et al. [13] proposed a unified approach for collecting data about smartphone interactions in an appropriate granularity for user modeling.

In this paper we report an investigation into implicit profiling and adaptive user interfaces for mobile news apps. First, a survey was conducted to examine news reading behaviour of users of mobile devices. A cluster analysis revealed three main types of mobile news reader characterized by five factors. Second, a study was conducted to investigate whether users of a news app could be identified in relation to the three types using a dedicated news app (Fig. 1), logging user's interactions during two weeks. Five characteristic factors were extracted from these logs and were used for training a classifier. Finally, a design

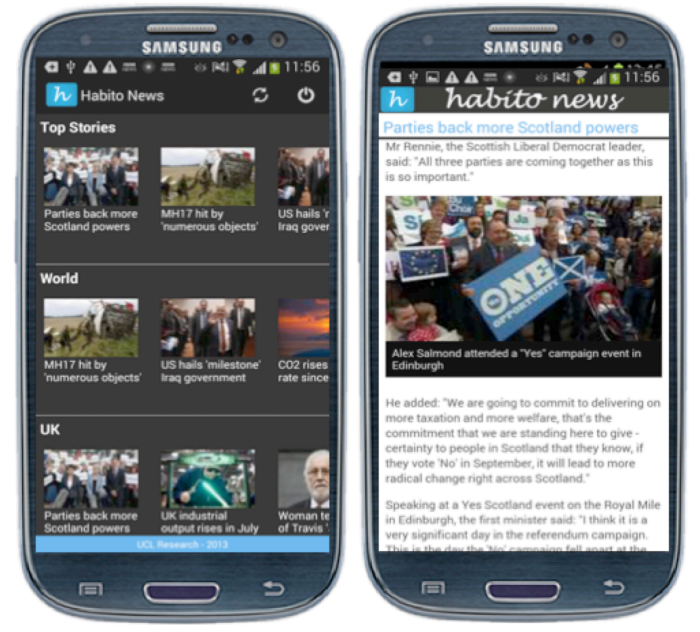

Fig. 1 Our BBC-mimic mobile news app that logs users' news reading interactions

of adaptive user interfaces for each of the three news reader types was evaluated. Our results suggest that different reader types would benefit from different user interfaces.

\section{IDENTIFICATION OF NEWS READER TYPES}

We deployed an online questionnaire ${ }^{1}$ using CrowdFlower with the aim of identifying stereotypical patterns of behaviour and individual experiences on mobile news reading. Although other studies [2,3] reported interesting insights on news access and consumption, this survey was designed to reveal reading and navigational behavior especially on smartphones.

The questionnaire consisted of 24 questions probing demographic information and news reading behaviour on mobile devices including the estimated time spent on news reading each day, the frequency of reading, browsing strategies, reading styles and so on. The sample comprised 140 respondents (54 females, $72 \%$ aged between 19-35, $60 \%$ hold a higher education qualification). The only requirement for participants was that they read news on smartphones. Respondents received a token payment for participating.

Analysis revealed interesting tendencies in users' preferences. Respondents mainly reported that they read the news once a day for between 10 and 30 minutes, preferably during the mornings and at home. Regarding their navigation and reading of news, no strategy dominates. When they browse, users do it either through all sections or they jump to a particular section whereas when they read they might skim or read the whole article.

In addition to the descriptive analysis we performed a hierarchical clustering analysis on the responses to all questions from all participants. The analysis revealed three homogeneity clusters and was conducted in three steps:

\footnotetext{
${ }^{1}$ Online questionnaire URL: http://goo.gl/HvoxBc
} 


\begin{tabular}{|c|c|c|c|}
\hline $\begin{array}{r}\text { Reader } \\
\text { types } \\
\text { Clustering } \\
\text { factors }\end{array}$ & 'Trackers' & 'Reviewers' & 'Dippers' \\
\hline Frequency & $\begin{array}{c}\text { Many times a } \\
\text { day }\end{array}$ & Once a day & $\begin{array}{c}\text { Less than once } \\
\text { a day }\end{array}$ \\
\hline $\begin{array}{c}\text { Total daily } \\
\text { reading }\end{array}$ & $5-10$ min & $10+$ min & $0-5$ min \\
\hline $\begin{array}{c}\text { Browsing } \\
\text { strategy }\end{array}$ & Both & $\begin{array}{c}\text { Through all } \\
\text { sections }\end{array}$ & $\begin{array}{c}\text { Particular } \\
\text { section }\end{array}$ \\
\hline $\begin{array}{c}\text { Reading style } \\
\text { Location }\end{array}$ & Skimming & $\begin{array}{c}\text { Detailed } \\
\text { reading }\end{array}$ & Scanning \\
\hline Transport & Home & Home \\
\hline
\end{tabular}

Table 1 News reader types for mobile news reading

(1) We first run a hierarchical clustering technique using Ward's cluster method to identify groups of users with the same characteristics. Three clusters were identified within the total data set; $31 \%$ in group A (Trackers), $36 \%$ in group $\mathrm{B}$ (Reviewers) and 33\% in group C (Dippers).

(2) We then used a Pearson's correlation test to associate questions with clusters. Five significant clustering factors were identified frequency; daily reading time; browsing strategy; reading style, and; location.

(3) We finally ran a cross tabulation statistical method to examine the distribution of the five factors over the clusters.

The three news reader types are summarized in Table 1 in relation to the five factors. Note that whilst the clusters are independent of each other, two clusters may not be discriminated on one factor, i.e., a user can belong to more than one cluster on that factor alone. We labeled the three news reader types as 'Trackers', 'Reviewers' and 'Dippers'.

Tracker: A person who likes to be informed about the latest stories and any updates to stories he or she is following, usually reading the news for up to 10 minutes at a time and several times a day at intervals, for example, when travelling. Due to her limited time she prefers to extract the important bits of a story (i.e. reading by skimming).

Reviewer: A person who likes to catch up on the day's news, preferably at home. He likes an in-depth analysis of the stories he reads and will read at length to fully understand the story (i.e. a detailed reading). He usually reads the news once a day, spending more than 10 minutes to get through all the stories of interest and likes being informed on a variety of topics.

Dipper: A person with a casual interest in the news but likes to read news on specific topics such as sport. She always knows what she is looking for so does not spend more than 5 minutes accessing the news. She likes to browse particular sections to find stories and looks for specific facts or pieces of information without reading everything (i.e. reading by scanning).
INTERACTION LOGGING AND CLASSIFICATION STUDY

Having characterised mobile news readers as one of three types, the question that follows is whether a news app could detect a user as being a particular reader type from their interactions alone. We now report a study to examine this question involving the development of a mobile news app capable of collecting and classifying users' interaction data.

\section{Habito News: the mobile news app}

We implemented an Android news app to log user's interactions whilst reading the news. To ensure its familiarity to users, our news app mimicked the BBC's app in terms of its visual presentation (Fig. 1). News stories are organised in rows of thumbnails and are retrieved from the BBC API using an asynchronous background task. To log the interactions we implemented a background service that unobtrusively collects data associated with general preferences and usage of the app, both navigational and reading, based on the five factors found in the survey study.

\section{Deployment and data collection}

The Habito News app was downloaded from Google Play and used by 23 participants (11 females) aged 22 to 33 years old $(\mathrm{M}=27, \mathrm{SD}=3.64)$ for two weeks. Participants were instructed to read the news, as they would do with any other available news app and to use it as their primary news app. After installing the app, users completed a form containing the same questions as contained in the survey. The app then recorded their interactions in relation to the five clustering factors in the survey (including precise scroll usage, reading time, frequency of reading, location, exact category scrolling event such as items browsed and swipe direction, etc). Although, other researchers conducted logging studies $[5,8]$, to the best of our knowledge none of them logged data in such fine granularity.

\section{User Modeling: Predicting News Reader Types}

The logged interaction data was used to identify each participant's news reader type using a naïve Bayes classifier. The classifier was developed in three steps:

(1) The five clustering factors were extracted from the raw interaction data by abstraction. For example, reading style was estimated using the formula ${ }^{2}$ in which the proportion of the article exposed to the user by using scroll positions, divided by the total size of the document and multiplied by the number of words to approximate the number of words viewed. The number of words read per $(w p m)^{3}$ was estimated by dividing the approximate number of words seen by the reading duration. Finally, the reading speed was interpreted as one of the three reading styles: reading for comprehension (i.e. detailed reading) up to $230 \mathrm{wpm}$; scanning, reading faster than $700 \mathrm{wpm}$, and; skimming, a reading speed between normal reading and scanning.

\footnotetext{
${ }^{2}$ words seen $=$ scroll_offset/scroll_range*no_of_words

${ }^{3} \mathrm{wpm}=$ words seen/reading duration
} 


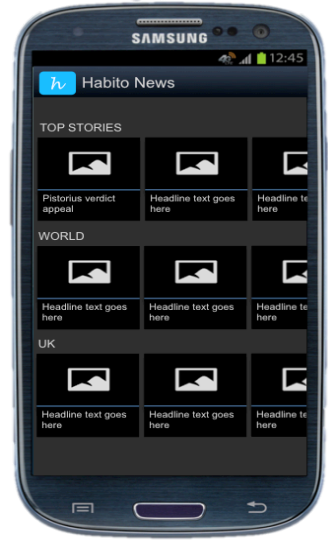

(a) Baseline

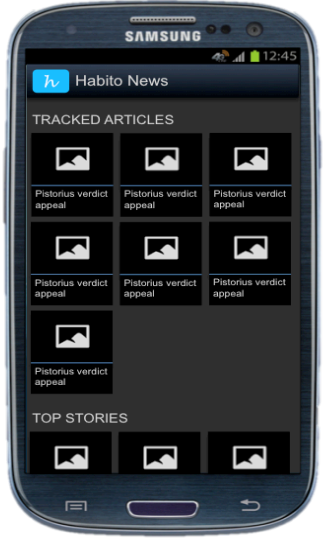

(b) Trackers

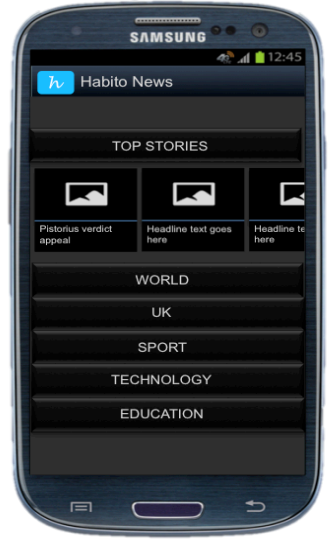

(c) Reviewers

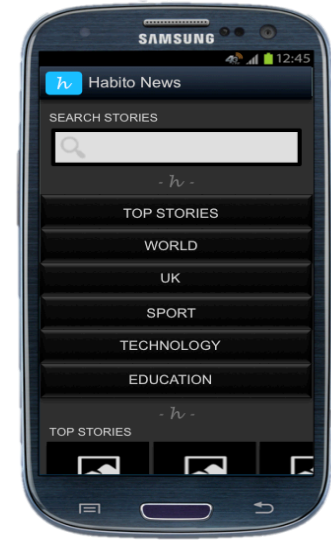

(d) Dippers

Fig. 2 The baseline interface with the adaptive variants for the three news reader type

Likewise, we extracted the frequency by keeping sessions of how frequent the user accessed our app, and whether the user accessed any other news app installed in their phones Regarding to the total daily reading time we added up the time spend on reading. Finally, we implemented another background service to capture user's location. To transform location (longitude, latitude) into public transport or home we used a heuristic formula in which stable consecutive location points were defined as home, and non-stable ones as public transport.

(2) Creating the training set: each user was classified by taking the transformed values of their interaction $\log$ for each of the five factors identified by the clustering of survey results (e.g. frequency, total daily reading, etc). We classified each user as a particular news reader type corresponding with the five factors and making the simplifying assumption that the factors are equally weighted. So, each item in the training set was of the following form: user id, $F_{1}, F_{2}, F_{3}, F_{4}, F_{5}$, Class (where Fn: Factor, Class: news reader type).

The training set therefore consisted of 23 users, each consisting of a profile of five factors derived from their interaction logs and each identified as one of the three types of reader. A word of caution is appropriate here. As a separate check of the classification of reader types, each user had been asked to complete the survey. Only a 55\% correspondence was found between participants' answers to the survey (e.g., for how long they read the news each day in total) and the collected interaction data. It was of course such self-assessments in the survey that produced the original clustering factors. Hence a separate study is needed to establish whether the same news reader types would emerge from the cluster analysis if logged interaction data were added to the survey data.

(3) Implementing the classifier: We used a canonical profile for each of the three types to validate the classifier. The canonical profiles represent the interaction log values for the five factors for a prototypical user of that reader type.
For example, for the Tracker reader, the canonical profile says they read news many times a day, with a total reading time of less than five minutes, using both browsing strategies, a skimming reading style, and primarily a public transport location.

The classifier was able to correctly classify the canonical profiles with up to $88 \%$ accuracy for Trackers, $91 \%$ for Reviewers and $92 \%$ for Dippers. Future work will validate the classifier over a new corpus of interaction logs to be collected from users of the news app.

\section{ADAPTIVE UI AND EVALUATION STUDY}

Having shown that we can recognise a user's news reader type from a data log of their interactions, the next question that arises is whether the different types would benefit from different adaptive forms of the news app.

To examine this question we devised adaptive user interfaces for each news reader type through a series of semi-structured interviews and contextual inquiries with 10 participants. The interviews probed participants' about their news reading and their opinions on a range of customizable features of current mobile news apps and design suggestions of our own. Participants were also asked to experience reading the news with our news app and provide comments on its design. For example, Trackers receive the latest stories or updates in the top static area for quick access and we replace the horizontal organisation of stories to a full-width layout because they like to get a quick snapshot of the news. For Reviewers we did not make significant changes because it seemed that the baseline interface almost meet their needs. For Dippers, we provide the search functionality to enable quick browsing of specific facts and the jump-to category feature to allow faster navigation. For Trackers and Dippers we also have the extra feature of summaries to support their reading behaviour. The way summary is presented in each of these types differs depending on their characteristics. For example, Trackers receive summaries as a paragraph because they are skimmers, whereas we display the 
summary as bullet points for Dippers who read in a faster pace. The adaptive features of each type are listed below:

(1) Adaptive UI for Trackers (Fig. 2b)

- Top static area for the tracked articles

- Full-width categories layout

- Option of a summarised version of the story

(2) Adaptive UI for Reviewers (Fig. 2c)

- No visuals (articles' thumbnails) in the menu structure, only top stories kept open

- Accordion to ease access categories

(3) Adaptive UI for Dippers (Fig. 2d)

- Search functionality

- Easy access to articles of a particular category (jump-to)

- Option of a summarised version of the story

\section{Evaluation Study of the Adaptive UI variants}

We conducted a laboratory study to compare the baseline and adaptive variant of the news app interface for each news reader type. The comparison was of time to find (browsing) and read (reading) the news in benchmark tasks. The following hypotheses were formulated:

$\mathbf{H}_{1}$ : The adaptive UI for Trackers improves their performance over the baseline UI.

$\mathbf{H}_{2}$ : The adaptive UI for Reviewers improves their performance over the baseline UI.

$\mathbf{H}_{3}$ : The adaptive UI for Dippers improves their performance over the baseline UI.

\section{Participants}

18 individuals (7 Trackers, 5 Reviewers and 6 Dippers) aged 20 to 30 years old $(\mathrm{M}=23, \mathrm{SD}=2.26)$ participated in the study. All participants had considerable experience in using mobile devices and particularly with Android OS.

\section{Materials}

We used Justinmind ${ }^{4}$ to develop the interactive wireframes (Fig. 2) and deployed them on a Samsung Galaxy S3 (4,8inch screen, $1280 \times 720$ resolution). A video camera was used to record participants' interactions with the device. A comparison questionnaire was used to measure their subjective preferences between the two interfaces. We asked participants to choose which interface they preferred (using a scale ranging from "Mostly A" to "Mostly B", where Mostly A means strong preference for interface A baseline- and Mostly B for interface B -adaptive-).

\section{Procedure}

At the beginning of each session participants completed a questionnaire that would allow their news reader type to be determined. Demographic information was also collected. Once their type was identified, participants were instructed to complete a set of predefined tasks on both interfaces. It should be noted that the tasks necessarily varied between news reader types, appropriate to their characteristic patterns of news reading. For example, Reviewers were

\footnotetext{
${ }^{4}$ http://www.justinmind.com/
}

asked to find and read specific articles and then briefly described what the article was about. After completing the tasks with both interfaces, participants completed the comparison questionnaire. A short debriefing at the end of the experiment sought participants' views on how easily they were able to find and read articles with each interface and the features of the adaptive variant.

\section{Design}

The experiment was a one way within-subject design on UI type (baseline, adaptive variant), conducted independently on each group of news reader type (Trackers, Reviewers, Dippers). Dependent variables were the time taken to find articles and the time taken to read them. Participants were not aware which interface was the baseline and which was the adaptive design.

\section{Results}

An independent-samples t-test was conducted to compare the time taken to find and read stories in the baseline and in the adaptive variant interface for each news reader type.

Baseline vs. Adaptive for Trackers: There was a significant difference in the scores for the time taken to find articles in the baseline $(M=21.42, S D=7.59)$ and the adaptive for Trackers $(\mathrm{M}=9.85, \mathrm{SD}=4.37)$ conditions; $\mathrm{t}(12), \mathrm{p}=0.004$. There was also a significant difference in the scores for the time taken to read articles in the baseline $(M=147.42$, $\mathrm{SD}=62.05)$ and the adaptive for Trackers $(\mathrm{M}=77$, $\mathrm{SD}=30.47)$ conditions; $\mathrm{t}(12) \mathrm{p}=0.019$. We therefore accept $\mathbf{H}_{1}$ - the adaptive interface for Trackers improved their performance over the baseline.

Baseline vs. Adaptive for Reviewers: There was not a significant difference in the scores for the time taken to find articles in the baseline $(\mathrm{M}=21.40, \mathrm{SD}=5.77)$ and the adaptive for Reviewers $(M=25, S D=10.12)$ conditions; $t(8)$, $\mathrm{p}=0.509$. No significant difference found in the scores for the time taken to read articles in the baseline $(M=199$, $\mathrm{SD}=12.04)$ and the adaptive for Reviewers $(\mathrm{M}=221.60$, $\mathrm{SD}=27.15)$ conditions; $\mathrm{t}(8) \mathrm{p}=0.127$. We therefore reject $\mathbf{H}_{2}$ - the adaptive interface for Reviewers did not improve their performance over the baseline.

Baseline vs. Adaptive for Dippers: There was not a significant difference in the scores for the time taken to find articles in the baseline $(\mathrm{M}=18.67, \mathrm{SD}=5.98)$ and the adaptive for Dippers $(M=25.50, S D=8.43)$ conditions; $t(10)$, $\mathrm{p}=0.137$. There was also not a significant difference in the scores for the time taken to read articles in the baseline $(\mathrm{M}=180.83, \mathrm{SD}=79.17)$ and the adaptive for Dippers $(\mathrm{M}=117.33, \mathrm{SD}=38.51)$ conditions; $\mathrm{t}(10) \mathrm{p}=0.108$. We therefore reject $\mathbf{H}_{3}-$ the adaptive interface for Dippers did not improve their performance over the baseline.

\section{Satisfaction and User Comments}

$71 \%$ of Trackers preferred the adaptive interface to browse with $29 \%$ of them stating a strong preference, whereas all of them found the adaptive interface better for reading. However, $80 \%$ of the Reviewers preferred the baseline for 
navigation, and $60 \%$ easier for reading. Dippers stated a neutral preference on both interfaces with $33 \%$ for each.

In their post-experiment comments, Trackers praised the possibility to switch between the full article and its summary, but also suggested extra features to support tracked articles, e.g. visually differentiating read articles and new ones. They however strongly disliked the menu structure; the vertical scrolling for categories, due to the fact they want to be able to browse all categories with ease.

Reviewers reported they did not find the adaptive menu beneficial and would prefer a snapshot of articles within multiple categories as opposed to being restricted to one. None reported wanting article summaries despite reading long articles and being probed in the post interviews.

Dippers also preferred article summaries and the option to switch between the full and the summarised version of an article. They praised the menu structure with the jump-to category but recommended a return-to-top functionality (widely common technique at websites navigation).

\section{DISCUSSION}

The key findings of these three studies are: (1) mobile news readers can be characterised within three types; (2) it is possible to detect a user's news reader type from analysis of their interactions with a news app, and; (3) different reader types benefit from different forms of news app interface.

We showed that mobile news readers can be distinguished as Trackers, Reviewers or Dippers according to their frequency of news reading, their duration of news reading, their browsing strategy, their reading style and their location. We showed that a Bayesian classifier can identify the user of a news app according to their news reader type from a log of interactions with a news app from which the clustering factors could be extracted. The evaluation study demonstrated that Trackers performed better with the adapted interface variant, whereas Reviewers and Dippers performed better with the baseline interface. Trackers also expressed a preference for the adapted variant created for them, whereas Reviewers and Dippers did not prefer the variants created for them. Those two variants were clearly not successful, however this does not preclude that successful forms could be created and further investigation is needed. However the more significant finding is that their needs and those of the Trackers are not met by the same interface and that adaptation is desirable, which can reliably been achieved by the system.

Based on evaluation findings, we modified the Reviewers interface and dropped the accordion style organisation to restore the horizontal rows organisation of the baseline interface. We also added the feature of expanding categories to provide the big picture of stories within a category. Likewise, we suggest a simpler and more straightforward accordion style organisation for improving
Dippers navigation, combining the jump-to category and the return-to-top functionality.

\section{CONCLUSION AND FUTURE WORK}

We explored the feasibility of recognising patterns of news reading interactions and evaluated three adaptive interface designs for different news reader types. We show that from their interaction log, a specific user can be recognised as one of three kinds. The reader types emerging from the online survey are well defined and distinct. The evaluation of the three variant interfaces suggests that different news reader types need different user interfaces. We have demonstrated a method for monitoring users' news reading behaviour and inferring news reader type from it.

In the future we will further explore the design of adaptive interfaces, in order to be in a position to demonstrate a complete adaptive mobile news framework providing automatic personalisation of news apps.

\section{REFERENCES}

1. Ofcom, News consumption in the $U K$, Public report (2014).

2. Pew Research Centre, The Future of Mobile News, Public report (2012).

3. Reuters Institute, Tracking the future of news, Public Report (2014)

4. Billsus, D. \& Pazzani, M. A hybrid user model for news story classification. Springer Vienna (1999), 99-108.

5. Billsus, D. \& Pazzani, M. Adaptive news access. In The adaptive web. Springer Berlin Heidelberg (2007).

6. Carreira, R., Crato, J. M., Gonçalves, D., \& Jorge, J. A. Evaluating adaptive user profiles for news classification. In ACM IUI 2004.

7. Gauch, S., Speretta, M., Chandramouli, A., \& Micarelli, A. User profiles for personalized information access. In The adaptive web. Springer Berlin Heidelberg (2007).

8. Grzeschik, K., Kruppa, Y., Marti, D., \& Donner, P. Reading in 2110-reading behavior and reading devices: a case study. The Electronic Library (2011).

9. Jameson, A. Adaptive interfaces and agents. HumanComputer Interaction: Design Issues, Solutions, and Applications, (2009).

10. Oulasvirta, A., Rattenbury, T., Ma, L., \& Raita, E. Habits make smartphone use more pervasive. Personal and Ubiquitous Computing (2012).

11. Tavakolifard, M., Gulla, J., Almeroth, K., Ingvaldesn, J., Nygreen, G. \& Berg, E. Tailored news in the palm of your hand: a multi-perspective transparent approach to news recommendation. In $A C M W W W 2013$.

12. Westlund, O. From mobile phone to mobile device: News consumption on the go. Canadian Journal of Communication (2008), 33(3).

13. Woerndl, W., Manhardt, A., \& Prinz, V. A framework for mobile user activity logging. In MUSE 2010. 